\title{
A Percepção dos Alunos de Química Orgânica sobre o Uso do Socrative em Aulas Síncronas na Universidade de Brasília (UnB)
}

\section{Maria Hosana Conceição', Olga M. Albuquerque¹, Lúcia Helena Soares e Silva ${ }^{2}$ e Lúcia Maria da Graça Rabelo ${ }^{1}$}

\author{
${ }^{1}$ Programa de Pós-Graduação em Propriedade Intelectual e Transferências de Tecnologia \\ para a Inovação (PROFNIT) - Universidade de Brasília (UnB) - Brasil | hosanac@unb.br; \\ olgamaria@unb.br ;lucia.graca@aluno.unb.br | https://orcid.org/0000-0002-0823-7841; \\ https://orcid.org/0000-0001-6824-603X; https://orcid.org/0000-0002-6316-9167 \\ 2Programa de Pós-Graduação em Botânica (PPGB) - Universidade de Brasília (UnB) - \\ Brasil | Lsoares@aluno.unb.br| https://orcid.org/0000-0001-6025-3201
}

\begin{abstract}
Resumo: No último ano, com a crise mundial da saúde pública, devido à pandemia da Covid-19, o ensino presencial deu lugar ao uso de plataformas tecnológicas para aulas online. Assim a Universidade de Brasília (UnB) preparou o seu corpo docente, para o ensino remoto, com a oferta de cursos sobre as plataformas do Moodle Aprender 3 e do Office 365 da Microsoft. Por outro lado, o planejamento das aulas, no modo remoto, exigiu de cada professor, uma sequência didática, lógica, para a participação dos estudantes, tanto nas aulas síncronas, quanto nas atividades assíncronas. Assim, o objetivo principal deste estudo foi conhecer a percepção dos estudantes da Farmácia sobre o uso da plataforma Socrative, nas aulas síncronas da disciplina de Química Orgânica. Trata-se de uma plataforma online na qual os estudantes praticam o conteúdo trabalhado em sala de aula, usando os seus smartphones ou tablets. O estudo contou com a participação de sessenta estudantes de segundo semestre do curso de Farmácia, da disciplina de Química Orgânica, ofertada no $1^{\circ}$ semestre de 2020. que realizaram as avaliações sobre a nomenclatura IUPAC dos compostos orgânicos. Dos 40 estudantes, que responderam ao questionário, foram consideradas apenas 34 respostas, na análise de conteúdo, com o uso do software IRAMUTEQ. O estudo mostrou-se promissor para o uso do Socrative nas categorias de vantagens, "dificuldades", sugestões e contribuições.
\end{abstract}

Palavras-chave: IRAMUTEQ; Socrative; Covid-19.

The Perception of Organic Chemistry Students on the Use of the Socrative as an Active Learning

Abstract. In the last year, with the global public health crisis, due to the Covid-19 pandemic face-to-face education gave way to the use of technological platforms for online classes. Thus, the University of Brasilia (UnB) prepared its teaching staff for remote teaching, offering courses on the Moodle Aprender 3 and Office 365 platforms from Microsoft. On the other hand, the planning of the classes, in the remote mode, demanded from each teacher, a didactic sequence, logical, for the participation of the students, both in the synchronous classes, as in the asynchronous activities. Thus, the main objective of this study was to know the perception of Pharmacy students on the use of the Socrative platform, in the synchronous classes of the discipline of Organic Chemistry. It deals with an online platform in which students practice the content worked in the classroom using their smartphones or tablets. The study had the participation of sixty students from the second semester of the Pharmacy course, of the discipline of Organic Chemistry, offered in the 1st semester of 2020 who carried out the evaluations on the IUPAC nomenclature of organic compounds. Of the 40 students who answered the questionnaire, only 34 responses were considered in the content analysis, using the IRAMUTEQ software. The study proved to be promising for the use of Socrative in the categories of advantages, "difficulties", suggestions and contributions.

Keywords: IRAMUTEQ; Socrative; Covid-19. 


\section{Introdução}

"No dia 12 de março de 2020, devido à situação de saúde pública pela pandemia da Covid-19, a Exma. Reitora da Universidade de Brasília (UnB), Profa. Márcia Abrahão assinou o Ato 0392/2020, suspendendo as atividades administrativas e acadêmicas presenciais e, constituiu o Comitê Gestor do Plano de Contingência da Covid-19 da UnB, Coes, composto por especialistas da Saúde, da Educação e da Administração, para pensar nas ações de enfrentamento da pandemia que culminaram na retomada das aulas no modo online/remoto" (Conceição et al., 2020, p. 247, no prelo). Imediatamente, formou-se uma rede, em que os professores, se mobilizaram para ajudar uns aos outros; "aqueles com mais experiência com as ferramentas do Moodle Aprender 3, por exemplo, passaram a capacitar outros colegas, mesmo sem conhecê-los pessoalmente". Segundo Conceição e Pires, "o novo formato motivou para a atualização das aulas clássicas do ensino presencial e, acrescentou que ainda não há a mensuração total dessa aprendizagem, mas é excepcional; não somente nós professores aprendemos mas os alunos também ganharam muito no processo de aulas online (Conceição \& Pires, 2020, p. 1). Diante desse novo formato de sala de aula virtual, foi necessário desenhar a organização dos conteúdos distribuidos em aulas síncronas e assíncronas, respectivamente, aquelas em que o professor e o aluno compartilham o mesmo espaço na plataforma escolhida e aquelas onde os estudantes realizam as tarefas ao seu tempo, sem a presença de outros colegas ou do professor (Prata, 2020).

Nos últimos anos, as ferramentas tecnológicas para o uso nas salas de aula, tanto tradicionais quanto virtuais (atuais) têm recebido a atenção dos educadores e pesquisadores do mundo todo (Choudhury et al., 2016; González, 2018; Ha, 2020; Herrada et al., 2020; Muir et al., 2020; Papadopoulos et al., 2018; Slipukhina et al., 2020). O sistema tecnológico denominado de Avabodhaka, um software com links destinado a realização de tarefas e download de arquivos, como por exemplo, documentos, áudios e vídeos e permitem que os estudantes postem as suas perguntas para o professor, de forma anônima e instantânea, foi utilizado por pesquisadores indianos, com boa aceitação pelos alunos. Um estudo empírico, com um grupo de estudantes, mostrou a boa aceitabilidade do Avabodhaka e identificou a necessidade de melhorias na latência do streaming das atividades que dependiam do aúdio (Choudhury et al., 2016).

Socrative é outro exemplo de software bastante utilizado, ultimamente, como apoio didático por professores de diversas áreas do conhecimento, podendo ser baixado tanto para o computador quanto para o smartphone, a partir do registo no site https://www.socrative.com/plans/\#login. A versão gratuíta nos permite trabalhar com uma sala de até 50 participantes. Para que os estudantes possam participar da atividade devem receber o código da sala, enviado pelo professor. O Socrative oferece uma ampla gama de possibilidades para criar tarefas e gerenciar as atividades. As tarefas podem ser questionário com questões do tipo verdadeiro ou falso; múltipla escolha ou ainda resposta curta; as quais podem ser gerenciadas no "ritmo do professor" ou no "ritmo do estudante". Na primeira escolha, o professor lança as questões e as controla da sua tela de tal forma que os estudantes as visualizem. Além disso, o professor tem a opção de inserir imagem para as perguntas e armazenar os questionários para compartilhar com equipes de trabalho (Cerqueiro \& Harrison, 2019). Cerqueiro e Harrison (2019) usaram o recurso de duas modalidades do Socrative; a "controlada pelo professor" e a de "games"; para avaliar 68 estudantes do curso de Licenciatura em Pré-Escola da Faculdade de Educação da Universidad de Castilla-La Mancha (UCLM/Toledo/España). Os resultados mostraram que as duas modalidades foram atrativas para os estudantes e colaboraram como uma ferramenta de avaliação formativa, principalmente, pelo feedback imediato.

Ainda em relação ao uso de plataformas tecnológicas, como uma forma de recurso didático, Christianson (2020) utilizou o Socrative, como uma ferramenta de aprendizagem ativa, na sala de aula online, nesse período de pandemia da Covid-19. Os comentários da avaliação dos estudantes indicaram que o uso do Socrative, para a aprendizagem de Química Geral, pode ser uma ferramenta útil na sala de aula remota e interativa. 
Ainda no contexto do uso do Socrative, Domingos e Recena (2010) realizaram uma pesquisa, com abordagem qualitativa, para trabalhar os conteúdos de funções orgânicas oxigenadas e nitrogenadas. As professoras utilizaram como instrumentos de coleta de dados a observação e a gravação, em vídeos, das aulas em que houve o desenvolvimento das atividades e dos materiais produzidos pelos estudantes. As apresentações seguiram com perguntas e respostas de cada vídeo, permitindo concluir que houve a oportunidade, para os alunos, de reestruturação dos esquemas de assimilação e acomodação no processo de ensino e aprendizagem, possibilitando a construção dos conhecimentos em química orgânica (Araújo Domingos \& Piazza Recena, 2010).

Este estudo teve o foco qualitativo. Tejedor (2020) considera a pesquisa qualitativa como uma pesquisa ativa, devido, principalmente, aos paradigmas, como interpretativo e o sócio-crítico. O primeiro está alicerçado às correntes humanisticas que se concentram nas análises dos significados das ações humanas e o segundo, o sócio-crítico, se encontra nas bases das escolas como, por exemplo, Frankfurt, Freire dentre outras (Tejedor, 2020, pp. 9-15).

A motivação da equipe baseou-se em dois pontos: explorar o uso do Socrative, como apoio didático aos estudantes da disciplina de Química Orgânica, nas aulas online, planejadas na sala virtual do Moodle Aprender 3 e conhecer a opinião dos estudantes sobre o uso dessa ferramenta tecnológica como recurso de avaliação formativa no período de aulas online.

\subsection{Justificativa}

A crise sanitária pela qual o Brasil e o mundo estão passando, devido à pandemia da Covid-19, fez com que as Universidades e os Centros de Ensino adequassem a oferta dos cursos com aulas no modo online/remoto. Assim, a Universidade de Brasilia (UnB), desde o mês de julho de 2020, vem se dedicando a capacitar os seus professores com a oferta de treinamentos nas plataformas do Moodle Aprender 3 e do Office 365 da Microsoft. Além da capacitação, o Centro de Educação à Distância (Cead) da UnB criou as Rotas de Inovação Universitária (RIU) que contém os recursos e as estratégias de aulas para o ensino não presencial (Leite, 2021). Portanto, com todo esse aparato de condições de trabalho para as aulas no modo online, esse projeto intitulado " $\mathrm{A}$ Percepção dos Alunos de Química Orgânica sobre o Uso do Socrative em Aulas Síncronas na Universidade de Brasília (UnB)" utilizou a plataforma, gratuita, Socrative como um recurso didático para as aulas de Química Orgânica online.

\section{Metodologia}

\subsection{Planejamento da Sala Virtual}

O aprimoramento do planejamento de uma sala de aula virtual exige dos professores conhecimento, dedicação e atualização das ferramentas tecnológicas. A pesquisa ocorreu, na sala virtual do Moodle Aprender 3, durante o $1^{\circ}$. semestre de 2020, em duas etapas: A $1^{\underline{a}}$ etapa, contou com a aplicação de quatro avaliações, entre os meses de setembro a novembro, para 60 estudantes do $2^{\circ}$. semestre do Curso de Farmácia, matriculados na disciplina de Química Orgânica Aplicada à Farmácia (QOAF). As avaliações foram preparadas, com o uso do Socrative (https://www.socrative.com/), no modo "Teste", com os conteúdos de hidrocarbonetos, alcooís, aldeídos, cetonas, ácidos carboxílicos e seus derivados. Cada Teste contou com até cinco perguntas, elaboradas nas opções de verdadeiro ou falso; múltipla escolha e resposta curta. Além disso, foi escolhido, na configuração do Socrative, o modo "controlado pelo professor". 
A $2^{a}$. etapa, ocorreu no mês de novembro e teve a finalidade de conhecer a percepção/opinião dos estudantes sobre o uso do Socrative. Para isso, foi preparado um Questionário, intitulado "Pesquisa Qualitativa Sobre o Uso da Plataforma Socrative", utilizando o aplicativo Microsoft Forms, do Office 365, contendo cinco perguntas, todas preparadas no modo de "Resposta curta", quais sejam:

1. qual a sua opinião sobre o uso da plataforma Socrative para a interação professoraluno no ambiente das aulas no modo remoto?;

2. Aponte as vantagens da plataforma Socrative, para o seu processo de formação, quanto ao reconhecimento das famílias dos compostos orgânicos na disciplina de Química Orgânica Aplicada à Farmácia 1 (QOAF1);

3. Qual foi a contribuição do uso da plataforma Socrative para o seu conhecimento e familiarização com os compostos orgânicos na disciplina de QOAF1?;

4. Descreva qual foi a sua principal dificuldade para manejar a plataforma Socrative durante a aula síncrona de QOAF1. Se você tiver mais de uma resposta, por favor, não deixe de descrevê-las;

5. O que poderia ser melhorado, na disciplina de QOAF1, quanto ao uso do recurso da Plataforma Socrative?

O Questionário foi configurado para ser respondido com o nome e matrícula ocultos e ficou disponível, como um link, na sala virtual do Moodle Aprender 3, por um período de 15 dias.

\subsection{Características da Pesquisa}

A pesquisa contou com a abordagem qualitativa com o foco no "reconhecimento da subjetividade, do simbólico e da intersubjetividade nas relações como partes integrantes da realidade social" (Minayo, 2016, p. 18). Em complemento, a pesquisa pode ser classificada como exploratória, por ter utilizado, como recurso didático, o Questionário com perguntas abertas e distribuídas em categorias motivacionais (Gil, 2018).

\subsection{Método e Estatísticas Textuais}

Os dados foram analisados por meio de análise de conteúdo categorial (Bardin, 1977). Para tanto, o software IRAMUTEQ (versão 0.7 alpha 2) foi utilizado como uma ferramenta para auxiliar nas etapas de tratamento e interpretação da análise de conteúdo, bem como recuperação de trechos de fala aderentes às temáticas abordadas. O uso do software IRAMUTEQ é indicado para a sofisticação de análises textuais (Camargo \& Justo, 2013). É um software livre baseado na linguagem estatística do software R, possibilitando cálculos de base estatística em material qualitativo (Ratinaud, 2014). Atualmente, possui dicionários ativos em diversas línguas como inglês, francês, espanhol e português.

O Corpus textual analisado foi codificado conforme orientações presentes em manuais oficiais do IRAMUTEQ (Camargo \& Justo, 2018; Salviati, 2017). Foi composto de 34 diferentes textos de língua portuguesa e contou com o total de 3.455 ocorrências de 841 diferentes formas lexicais. Optou-se pela lematização do corpus textual, ou seja, o software realizou o processo de deflexionar as formas lexicais para determinar lemas. Assim, formas como "gostei", "gostaria" e "gostaríamos" foram consideradas diferentes ocorrências da forma "gostar" (Camargo \& Justo, 2013). Destaque-se que foram adicionadas como ativas na análise apenas formas nominais e adjetivas para processamento via IRAMUTEQ. Tal escolha se deu em atenção à necessidade de maior relevância semântica para o entendimento do contexto. As demais formas permaneceram como suplementares. 
Foi realizada uma análise de similitude para compreender o modo como se agruparam as diferentes percepções dos estudantes sobre a plataforma Socrative. Essa análise possui suas bases na teoria dos grafos e, no contexto do IRAMUTEQ, apresenta um grafo que demonstra a forma como as palavras de um determinado corpus textual se relacionam (Salviati, 2017). Além disso, apresenta as comunidades de palavras presentes em diferentes temáticas. Para a análise de similitude, foram consideradas as 50 palavras mais frequentes no corpus analisado. A contabilização de frequência foi realizada pelo próprio software IRAMUTEQ, variando da forma "questão" com frequência 65 e "silêncio" com frequência 3.

Após, foram selecionados diferentes trechos de fala como evidências empíricas de interpretações postas. Tais trechos foram selecionados conforme aderência à categoria de discussão e presença das principais palavras de cada temática representada. A partir da interpretação da análise de similitude foi possível compreender com profundidade o conteúdo derivado das percepções dos alunos sobre vantagens, contribuições e dificuldades relacionadas ao uso do Socrative. Assim como agrupar sugestões de melhoria para o uso do software em aulas virtuais futuras. Finalmente, organizou-se uma tabela que consolida os trechos de fala escolhidos para representar as evidências empíricas das interpretações postas no presente estudo. A Fig. 1 ilustra as etapas da pesquisa.
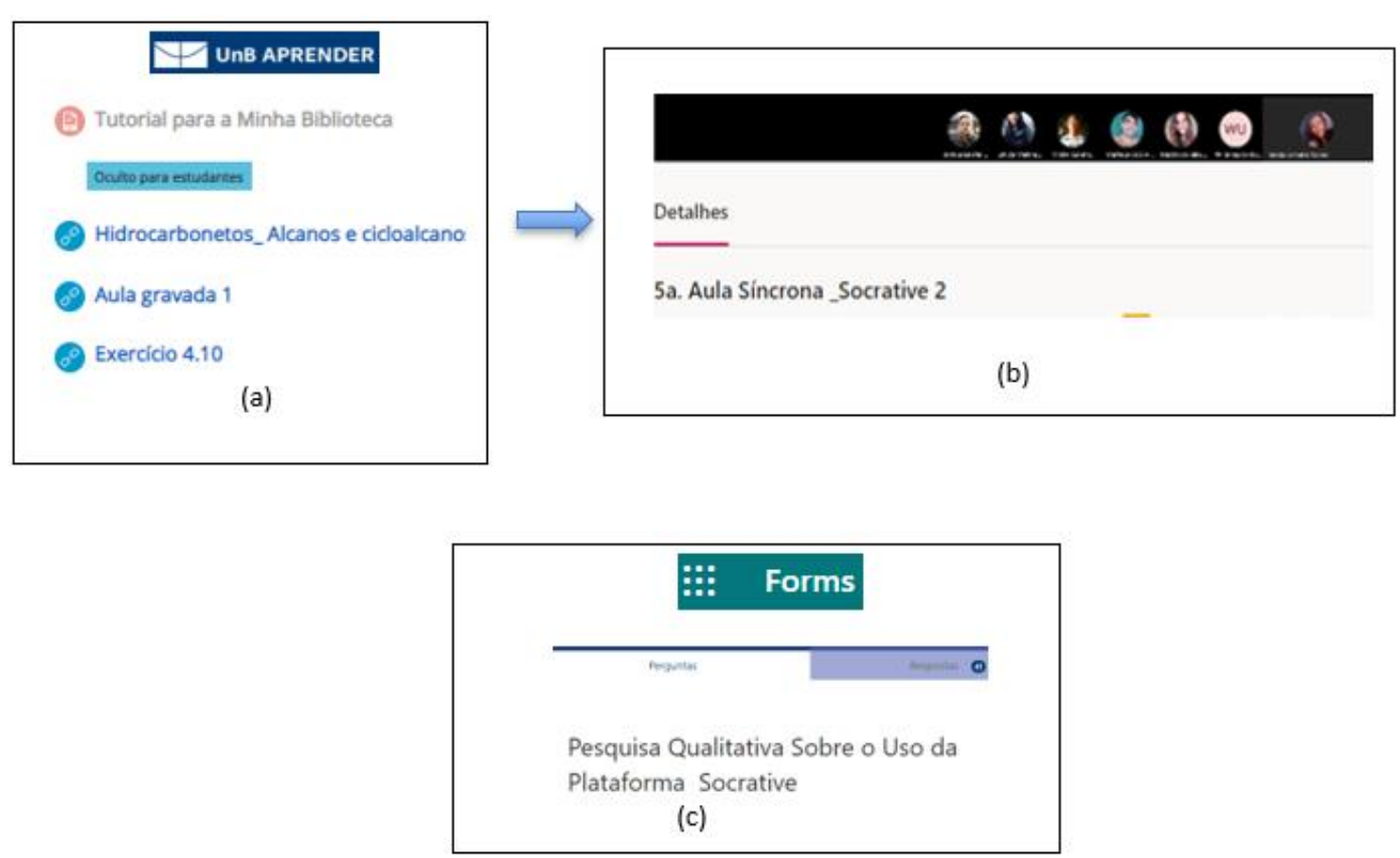

Fig. 1. Esquema ilustrativo das etapas do método do estudo: (a) orientação do estudo assíncrono no Aprender 3; (b) exemplo da aula síncrona com o uso do Socrative; (c) Aplicação do Questionário para conhecer o grau de satisfação dos estudantes com o Socrative. 


\section{Resultados}

\subsection{Análise de similitude}

A análise de similitude representada na Fig. 2 revela as diferentes temáticas tratadas no corpus textual analisado. De início, as comunidades de palavras destacadas em lilás e amarelo na análise de similitude ressaltam as vantagens percebidas pelos estudantes com o uso do Socrative em exercícios no contexto virtual.

A comunidade lilás possui ênfase nas vantagens relacionadas à realização de exercícios pelo Socrative, enquanto a comunidade amarela foca as vantagens em relação à dinâmica das aulas síncronas em geral. Diversos foram os relatos apontando a dinamicidade e diferenciação da plataforma, assim como facilitação do aprendizado, melhor assimilação de conteúdo e melhor interação em aulas síncronas. Tais resultados reforçam a importância do uso de ferramentas dinâmicas para avaliação em aulas no contexto virtual, haja vista que essas podem potencializar o interesse e participação dos estudantes nas atividades.

As dificuldades percebidas pelos estudantes estão demonstradas na comunidade de palavras destacada em verde. Nesse caso, revelaram-se duas discussões distintas. Primeiramente, diversos foram os relatos de estudantes que não apontam quaisquer dificuldades e ressaltam o potencial do Socrative em contribuir com a fixação do conteúdo da disciplina, em especial no que se refere a nomenclaturas e compostos. Porém, outros relatos ressaltam dificuldades de acesso devido a conexões ruins à internet, problemas com o próprio computador e erros advindos do uso conjunto do Socrative e a plataforma utilizada pelo professor para realização da aula síncrona.

Como consequências diretas das dificuldades percebidas, as comunidades de palavras destacadas em vermelho e azul escuro focam as sugestões postas pelos estudantes para melhor utilização da plataforma no futuro. Relatos apontam a necessidade de disponibilização de mais tempo para resposta das questões colocadas nos exercícios, assim como silêncio dos colegas e do próprio professor na condução da atividade durante aulas síncronas. Essa última sugestão foi colocada, haja vista a necessidade de concentração para realizar os exercícios. Ainda, os estudantes sugeriram que fossem realizados testes inicias, sem o propósito de atribuição de notas, com o objetivo de ambientação no Socrative. Não obstante, foi destacado o papel da plataforma em possibilitar a aproximação de aluno e professor no contexto virtual.

A comunidade azul claro apresenta a percepção dos estudantes sobre contribuições relacionadas ao uso da plataforma Socrative. Algumas contribuições colocadas foram a facilitação no conhecimento de nomenclaturas, fixação do conteúdo apresentado na aula e possibilidade de aprendizado com a análise de questões certas e erradas de forma mais interativa. Avalia-se que os estudantes apresentaram percepções positivas sobre o uso do Socrative em ambiente virtual. As dificuldades e sugestões relatadas não se referem diretamente à plataforma, mas sim a adaptações na dinâmica de aula que podem facilmente ser realizadas. 
Vol. 7 | Investigação Qualitativa em Educação: Avanços e Desafios

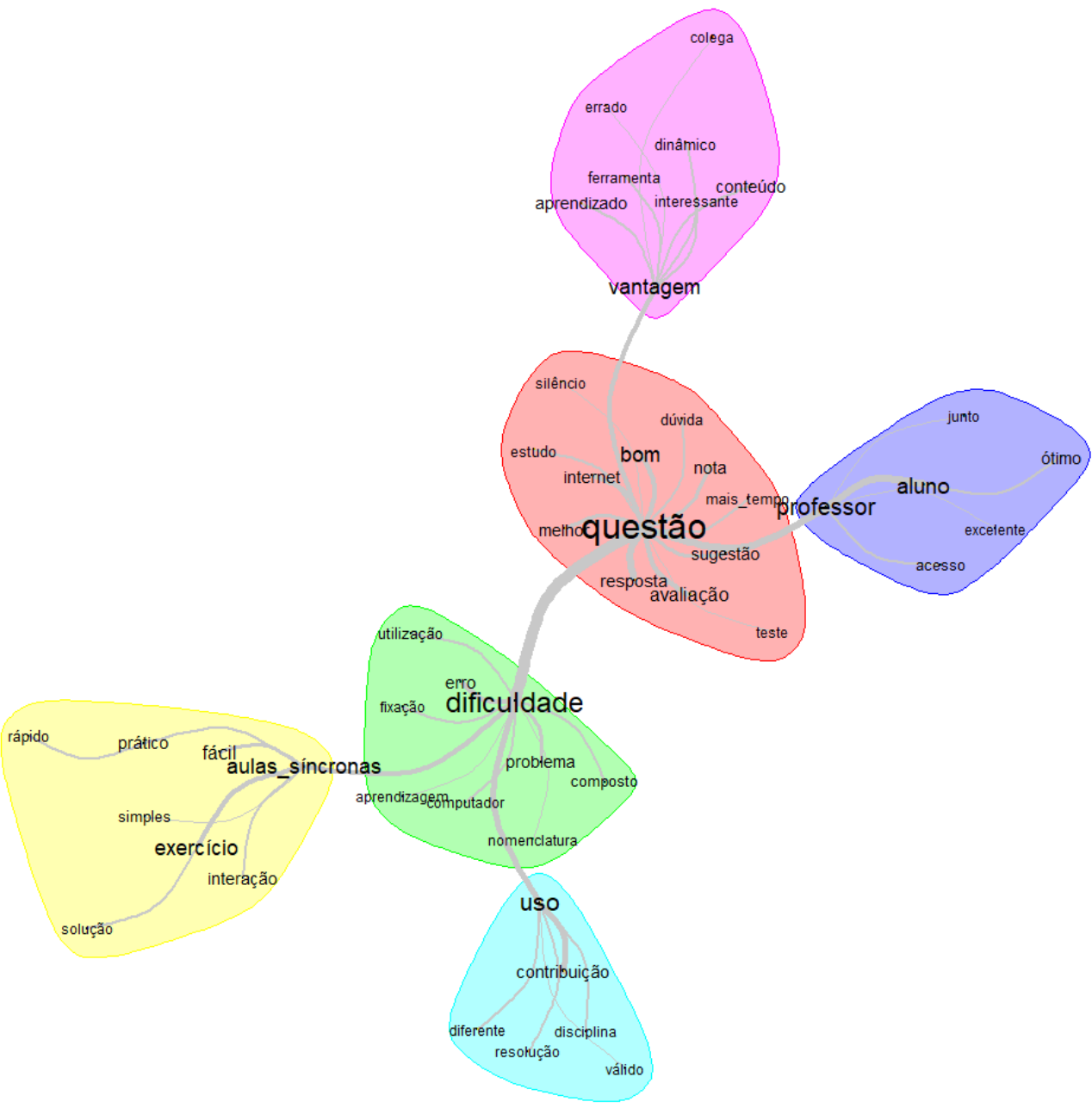

Fig. 2. Análise de similitude.

De modo a complementar a análise dos resultados de similitude, a Tabela 1 ilustra os trechos de falas selecionados como evidências empíricas. Nessa compilação, foram considerados o contexto da categoria a que se refere as falas. Foi possível observar que a plataforma Socrative se apresenta como uma opção para a interação entre estudantes e professor no contexto de aulas virtuais; além de ser uma ferramenta com forte potencial de aumentar o interesse dos estudantes durante aulas síncronas.

Tabela 1. Trechos das falas dos estudantes distribuídos em categorias e evidência empírica

\begin{tabular}{ccc}
\hline Categoria & Estudante & Evidência Empírica \\
\hline Vantagens & 7 & $\begin{array}{c}\text { As vantagens são que os exercícios } \\
\text { ajudam a solidificar os conhecimentos } \\
\text { adquiridos por meio da leitura e vídeo } \\
\text { aulas. }\end{array}$ \\
$\begin{array}{c}\text { As vantagens são a facilidade no } \\
\text { acesso e a resolução de forma } \\
\text { síncrona com os colegas. }\end{array}$ \\
\end{tabular}




\begin{tabular}{|c|c|c|}
\hline Categoria & Estudante & Evidência Empírica \\
\hline & 22 & $\begin{array}{l}\text { As vantagens do uso do Socrative se } \\
\text { referem ao fato de ser rápido, prático e } \\
\text { dinâmico. A apresentação das } \\
\text { estruturas fica muito mais interessante. }\end{array}$ \\
\hline & 32 & $\begin{array}{l}\text { Possibilita inserir um aspecto moderno } \\
\text { na maneira de avaliar. }\end{array}$ \\
\hline \multirow[t]{4}{*}{ Dificuldades } & 9 & $\begin{array}{l}\text { Não houve dificuldade no manejo, pois } \\
\text { é uma plataforma fácil de manejar. }\end{array}$ \\
\hline & 13 & $\begin{array}{l}\text { A única dificuldade que eu tive foi } \\
\text { enquanto não tinha computador porque } \\
\text { eu assistia as aulas síncronas pelo } \\
\text { celular e quando tinha socrative não } \\
\text { conseguia ouvir a professora. }\end{array}$ \\
\hline & 14 & $\begin{array}{l}\text { Minha maior dificuldade foi conseguir } \\
\text { focar nos exercícios e ao mesmo } \\
\text { tempo escutar a professora. Como tem } \\
\text { tempo, precisamos de concentração. }\end{array}$ \\
\hline & 33 & $\begin{array}{l}\text { Acredito que a minha dificuldade não } \\
\text { esteja ligada à plataforma. Foi mais por } \\
\text { problemas nessas últimas semanas } \\
\text { que tive com minha rede de internet. }\end{array}$ \\
\hline \multirow[t]{4}{*}{ Sugestões } & 10 & $\begin{array}{l}\text { Como sugestão de melhoria, poderia } \\
\text { revisar as questões dos exercícios e } \\
\text { aumentar o tempo de realização. }\end{array}$ \\
\hline & 18 & $\begin{array}{c}\text { A professora poderia não se comunicar } \\
\text { com os alunos a partir do momento que } \\
\text { abrisse a questão até o fechamento da } \\
\text { mesma. }\end{array}$ \\
\hline & 20 & $\begin{array}{l}\text { Uma sugestão é usar o Socrative com } \\
\text { tempo específico, não sendo } \\
\text { concomitante às aulas síncronas. }\end{array}$ \\
\hline & 24 & $\begin{array}{l}\text { Poderíamos ter mais tempo para } \\
\text { responder algumas questões. }\end{array}$ \\
\hline \multirow[t]{4}{*}{ Contribuições } & 1 & $\begin{array}{l}\text { Uma contribuição do uso do Socrative } \\
\text { foi o conhecimento de nomenclatura. }\end{array}$ \\
\hline & 8 & $\begin{array}{l}\text { A contribuição do uso do Socrative foi a } \\
\text { fixação do conteúdo estudado. }\end{array}$ \\
\hline & 17 & $\begin{array}{c}\text { Resolver as questões propostas com o } \\
\text { auxílio da professora, o que me fez } \\
\text { melhorar nas questões que tenho } \\
\text { dificuldade. }\end{array}$ \\
\hline & 29 & $\begin{array}{l}\text { A contribuição foi o aprendizado, pois } \\
\text { analisando quais são as respostas } \\
\text { corretas aprendemos mais. }\end{array}$ \\
\hline
\end{tabular}




\section{Conclusões}

As duas características mais atraentes do Socrative são pela facilidade de uso, tanto para os professores quanto para os estudantes, bem como pelo fato de ser gratuito e poder ser baixado no smartphone, no tablet ou no laptop. O estudo mostrou-se promissor para o uso do Socrative como uma ferramenta de avaliação formativa dos estudantes matriculados na disciplina de Química Orgânica. Por outro lado, devemos tomar o cuidado de oferecer essa ferramenta, também, no modo assíncrono, para permitir que todos os estudantes possam realizar a avaliação, sem ter prejuízos causados por eventuais problemas como, por exemplo, a falta de internet ou da disponibilidade do computador, no momento da aula síncrona. Dessa forma, o Socrative poderá ser previamente configurado para uma atividade assíncrona, com o tempo necessário para receber as respostas e dar o feedback. O intuito é que a plataforma Socrative funcione potencializando a interação e dinamicidade das aulas e evitando o eventual distanciamento de estudantes em diferentes condições de acesso. $O$ contexto pandêmico atual pede por novas dinâmicas, bem como novas formas de avaliação no ensino remoto e a utilização da plataforma Socrative apresentou potencial em manter o interesse dos estudantes participantes da presente pesquisa. No entanto, apesar das potencialidades do uso do Socrative em aulas virtuais, há se de considerar as diferenciações em termos materiais e até mesmo sociais da turma em vias de garantir a acessibilidade de todos.

\section{Referências}

Araújo Domingos, D.C., \& Piazza Recena, M.C. (2010). Elaboração de jogos didáticos no processo de ensino e aprendizagem de química: A construção do conhecimento. Ciências \& Cognição, $15,272-281$.

Bardin L. (1977). Analyse de contenu. Presses Universitaires de France, 1977.

Camargo, B. V., \& Justo, A. M. (2013). IRAMUTEQ: Um software gratuito para análise de dados textuais. Temas em Psicologia, 21(2), 513-518. https://doi.org/10.9788/TP2013.2-16

Camargo, B. V., \& Justo, A. M. (2018). Tutorial para uso do software IRAMUTEQ. Laboratório de Psicologia Social da Comunicação - $\quad$ UFSC, $1-32$. http://www.iramuteq.org/documentation/fichiers/Tutorial IRaMuTeQ em portugues_17.03.2016.pdf

Cerqueiro, F.F., \& Harrison, A. M.-M. (2019). Socrative in higher education: Game vs. other uses. Multimodal Technologies and Interaction, 3(3). https://doi.org/10.3390/mti3030049

Cerqueiro, Fátima Faya, \& Harrison, A. M. M. (2019). Socrative in higher education: Game vs. other uses. Multimodal Technologies and Interaction, 3(3). https://doi.org/10.3390/mti3030049

Choudhury, N., Venkatesh, T., Bhattacharya, S., \& Sarma, S. (2016). Avabodhaka: A System to Analyse and Facilitate Interactive Learning in an ICT Based System for Large Classroom. Procedia Computer Science, 84, 160-168. https://doi.org/10.1016/j.procs.2016.04.082

Christianson, A. M. (2020). Using Socrative Online Polls for Active Learning in the Remote Classroom. Journal of Chemical Education, 97(9), 2701-2705. https://doi.org/10.1021/acs.jchemed.0c00737

Conceição, M. H., Bocca, A. L., Brambatti, L. P., Silva, L. J. da, Cantarino, L., \& Costa, I. I. da. (2020). Ensino, pesquisa, extensão e saúde mental no contexto da crise sanitária da COVID19: o caso da Universidade de Brasília - UnB. In R. Moura (Ed.), Psicologia clínica e cultura contemporânea (pp. 243-260). https://doi.org/10.24824/978655868899.0

Conceição, M. H., \& Pires, C. (2020, March). Com criatividade e inovação, docentes reinventam ensino e pesquisa na pandemia. UnB Notícias, 1. http://noticias.unb.br/67-ensino/4584-comcriatividade-e-inovacao-docentes-reinventam-ensino-e-pesquisa-na-pandemia

Gil, A. C. (2018). Como elaborar projetos de pesquisa (Editora Atlas (ed.); 6a.). https://integrada.minhabiblioteca.com.br/\#/books/9788597012934/cfi/6/10!/4/2@0:0 
González, A. (2018). Turning a traditional teaching setting into a feedback-rich environment. International Journal of Educational Technology in Higher Education, 15(1). https://doi.org/10.1186/s41239-018-0114-1

Ha, M.-J. (2020). Implementing mobile-phone-based student response system in a large $\begin{array}{llll}\text { undergraduate } & \text { course. } & \text { 122-139. }\end{array}$ https://www.scopus.com/inward/record.uri?eid=2-s2.085090691670\&partnerlD $=40 \&$ md5=385a6192b56faa502bdb1b269349620c

Herrada, R. I., Baños, R., \& Alcayde, A. (2020). Student response systems: A multidisciplinary analysis using visual analytics. Education Sciences, 10(12), 1-23. https://doi.org/10.3390/educsci10120348

Leite, L. L. (2021). Rotas de Inovação Universitária - RIU. Centro de Educação à Distância Da Universidade de Brasília (Cead/UnB). https://riu.cead.unb.br/orientacoes

Minayo, M. C. de S. (2016). Fundamentos, percalços e expansão das abordagens qualitativas. In U. de A. Raimunda Silva, Universidade de Fortaleza; Simone Gonçalves de Assis, Fundação Oswaldo Cruz; Teresa Maria Bittencourt da Cruz, Universidade de Aveiro; Ronaldo Nunes Linhares, Universidade Tiradentes e Antònio Moreira (Ed.), Investigação Qualitativa: Inovação, Dilemas e Desafios (1a., pp. 17-48). Ludomedia. https://www.ludomedia.pt/prod_details.php?id=147\&catld=13\&offset=0

Muir, S., Tirlea, L., Elphinstone, B., \& Huynh, M. (2020). Promoting Classroom Engagement Through the Use of an Online Student Response System: A Mixed Methods Analysis. Journal of Statistics Education, 28(1), 25-31. https://doi.org/10.1080/10691898.2020.1730733

Papadopoulos, P. M., Natsis, A., \& Obwegeser, N. (2018). Using the students' levels of preparation and confidence as feedback information in quiz-based learning activities. In Communications in Computer and Information Science (Vol. 865, pp. 97-115). https://doi.org/10.1007/978-3319-94640-5_5

Prata, D. N. (2020). Assíncrono vs. Síncrono no Contexto da Educação Superior Pós-Pandemia (Rotas de Inovação Universitária (RIU)). https://riu.cead.unb.br/orientacoes/2-publicacoes/76sincrono-assincrono

Slipukhina, I., Chernetckiy, I., Kurylenko, N., Mieniailov, S., \& Podlasov, S. (2020). Applied aspects of instrumental digital didactics: M-learning with the use of smartphone sensors. CEUR Workshop Proceedings, 2740, 173-187. https://www.scopus.com/inward/record.uri?eid=2s2.0-85096419218\&partnerID=40\&md5=580f4098406a9d44f46d628957781c67

Tejedor, F. J. (2020). La Práctica de la investigación cualitativa: ejemplificación de estudios (Ludomedia (ed.); 1a., pp. https://www.ludomedia.pt/es/prod_details.php?id=151\&catld=0\&offset=0 